Research Article

\title{
Improving Camper Comfort by Optimizing the Vibration Isolation of Air-Conditioning Compressor
}

\author{
Hui Shi, ${ }^{1}$ Wenku Shi $\mathbb{D},{ }^{1}$ Liang Sun, ${ }^{2}$ and Zhiyong Chen ${ }^{1}$ \\ ${ }^{1}$ State Key Laboratory of Automotive Simulation and Control, Jilin University, Changchun 130022, China \\ ${ }^{2}$ Xuzhou Heavy Machinery Co., Ltd., Xuzhou 221004, China \\ Correspondence should be addressed to Wenku Shi; shiwk@jlu.edu.cn
}

Received 9 August 2020; Revised 17 October 2020; Accepted 23 November 2020; Published 24 December 2020

Academic Editor: Bekir Sahin

Copyright $\odot 2020$ Hui Shi et al. This is an open access article distributed under the Creative Commons Attribution License, which permits unrestricted use, distribution, and reproduction in any medium, provided the original work is properly cited.

Compressors are the main excitation source of vibration and noise in camper air-conditioning systems, which directly affects the $\mathrm{NVH}$ characteristics in campers. The vibration and noise reduction for camper comfort improvement can be implemented by the optimization method. This paper presents vibration and noise improvement in camper by systematically optimizing the vibration isolation performance of air-conditioning compressor. To analyze the compressor vibration characteristics, operational vibration tests under different conditions and modal identification are implemented. Based on the analysis results, a genetic algorithm is used to optimize the stiffness of the compressor vibration isolation element by the energy decoupling method (EDM). The stiffness, natural frequencies, and energy decoupling rate (EDR) of the compressor vibration isolation system (CVIS) are compared between preoptimization and postoptimization. The stiffness and strength characteristics of the optimized isolation system are verified by the finite element method. Operational vibration and noise tests of a camper are performed to validate the optimization effect. The improvement of camper comfort demonstrates that the proposed optimization procedure of CVIS is effective.

\section{Introduction}

With the rapid development of the social economy and improvements in road and transportation infrastructure, campers have become a popular choice for high-end selfdriving travel. However, the using comfort of campers can be largely affected by the NVH characteristics, which is mainly caused by air-conditioning systems (ACSs), especially for trailer-type campers; therefore, much research is required to reduce the NVH of ACSs for improving camper comfort, which is mainly caused by the compressors.

The excitation forces of compressor to the air-conditioning system are very complex. On one hand, the compressor produces the gas force, the friction force between the roller and cylinder, and inertia force of unbalanced mass of shaft during the variation of cylinder working volume; on the other hand, the compressor is driven by permanent magnet synchronous motor, for which the electromagnetic vibration is brought on by the radial force, cogging torque, pulsating torque, and inverter harmonics during operational vibration measurement. The excitation forces is correlated with both the electromagnetic and the mechanics. The vibration characteristics of the camper air-conditioning system are different from other vibration systems. Therefore, some different points in the vibration and noise investigation are considered.

For the NVH improvement research of ACSs with horizontal rolling rotor compressor, many scholars have carried out much work to reduce the vibration and noise level of ACSs by using lots of methods. In [1, 2], to analyze the sources of $\mathrm{NVH}$, the excitation forces such as gas force, friction force, and eccentric mass inertia force are analyzed. The natural characteristics [3] by the finite element method (FEM), vibration and noise response $[4,5]$, and unbalance dynamics of the rotor system by rotor dynamics [6] are also studied. All the above research on vibration attenuation of compressor is mainly on the natural characteristics analysis by FEM, source property analysis, and vibration response 
analysis, which require redesigning the compressor. Due to the complexity of a compressor system, redesigning efforts are relatively daunting; the camper vibration isolation system (CVIS) is to reduce the vibration transmissibility of compressor. Therefore, the vibration response caused by the compressor can be decreased by the optimization of CVIS, which costs less than redesigning compressors. Seve [7], Kim [8], and Li [9] et al. studied the stiffness, shape, and material improvement of the vibration isolation element. Li [10] and $\mathrm{Li}$ [11] et al. studied the natural frequencies and damping optimization of a CVIS. Dong [12] and Wang [13] studied different methods of dynamic modeling and transmissibility optimization, but the above study was only conducted from a certain aspect, and there was no systematic and comprehensive optimization study. Usually, for a CVIS optimization, three functions can be selected as optimization objective functions, i.e., minimum vibration transmissibility, minimum reaction force, and maximum decoupling rate of the vibration isolation system $[14,15]$. For minimum vibration transmissibility, it costs much effort to minimum transmissibility of all the key DoFs. For minimum reaction force, it is difficult to calculate the reaction force because it needs further the torque of CVIS. For maximum decoupling rate, it can be realized by elasticity center method, stiffness matrix method, and energy decoupling method (EDM). The elasticity center method enforces the mass center of CVIS to coincide with the spacial elasticity center, which is practically difficult. The stiffness matrix method is difficult to diagonalize both the mass matrix and stiffness matrix for practical limitation. The EDM can be applied at any coordinate system avoiding the practical limitations and is widely used. So, the EDM is commonly selected as the objective function.

In this research, the investigated ACS is shown in Figure 1. The vibration isolation performance of CVIS is optimized to improve the camper comfort by proposing an optimization procedure based on EDM. The optimization method based on energy decoupling rate (EDR) of CVIS is proposed to improve the vibration and noise level of camper. Then, an operational vibration test and modal identification test are performed to troubleshoot the vibration and noise level in the camper. Next, based on the energy decoupling method, a genetic algorithm is adopted to optimize the stiffness and EDR of the CVIS, and the vibration isolation element is redesigned as a large-damping pure rubber structure. After that, the strength and stiffness of the vibration isolation rubber element is verified by finite element simulation; finally, operational vibration and noise tests are performed in a camper to verify the effectiveness of the optimization.

\section{Optimization Method}

2.1. Dynamic Energy Decoupling Method. Usually, different DoFs in a CVIS are coupled, which widens the resonance frequency band and increases the likelihood of resonance. Therefore, the vibration and noise level can be effectively improved by increasing the EDR of different DoFs in the CVIS. The current decoupling methods include the elastic center method, stiffness matrix method, and energy

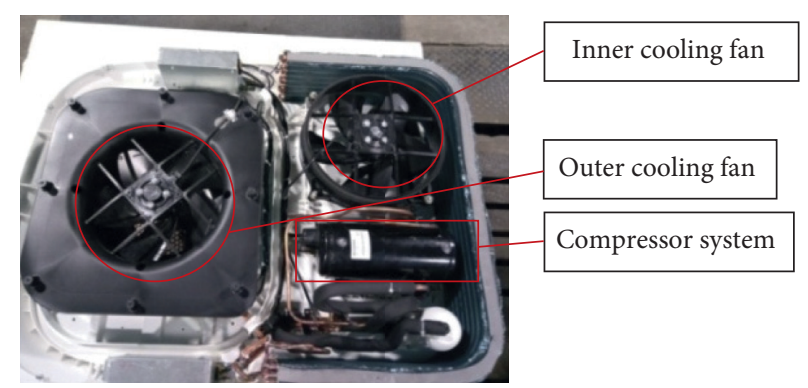

FIGURE 1: Air-conditioning system with rolling rotor compressor.

decoupling method [16]. Compared with the former two methods, the energy decoupling method is not constrained by the system's structural scale, installation position, and angle, so the energy decoupling method is selected for this research.

Due to vibration coupling, for a certain mode, the excitation force (moment) exerted on a system will be converted into kinetic energy and potential energy in the generalized coordinate system, thereby aggravating the system vibration. The total kinetic energy of the vibration system in the $i^{\text {th }}$ mode can be expressed as

$$
\begin{aligned}
E_{\max }^{i} & =\frac{1}{2} \dot{Q}_{\max }^{T} M \dot{Q}_{\max } \\
& =\frac{1}{2} \omega_{i}^{2} \sum_{k=1}^{6} \sum_{l=1}^{6} M_{k l}\left(\varphi_{i}\right)_{k}\left(\varphi_{i}\right)_{l}=\sum_{k=1}^{6} E_{k}^{i} .
\end{aligned}
$$

In equation (1), $\omega_{i}$ is the natural frequency of the $i^{\text {th }}$ mode; $M_{k l}$ is the element of the $k$ row and $l$ column in the system mass matrix; and $\left(\varphi_{i}\right)_{k}$ and $\left(\varphi_{i}\right)_{l}$ are the $k$ and $l$ elements of the $i^{\text {th }}$ mode, respectively; $i, k$, and $l=1,2, \ldots, 6$.

The kinetic energy in the $i^{\text {th }}$ mode of the $k^{\text {th }}$ generalized coordinate system is

$$
E_{k}^{i}=\frac{1}{2} \omega_{i}^{2} \sum_{l=1}^{6} M_{\mathrm{kl}}\left(\varphi_{i}\right)_{k}\left(\varphi_{i}\right)_{l} .
$$

Combining equations (1) with (2), the total vibration kinetic energy of the $i^{\text {th }}$ mode can be obtained by superposing the six component kinetic energies $E_{k}^{i}$. According to the energy conservation law, when there is more kinetic energy distributed in one direction of the $i^{\text {th }}$ mode, there will be less kinetic energy in other directions.

Therefore, the ratio of kinetic energy in the $k$ direction to the total kinetic energy of the system can be expressed as

$$
T_{k i}=\frac{\sum_{l=1}^{6} M_{k l}\left(\varphi_{i}\right)_{l}\left(\varphi_{i}\right)_{k}}{\sum_{k=1}^{6} \sum_{l=1}^{6} M_{k l}\left(\varphi_{i}\right)_{k}\left(\varphi_{i}\right)_{l}} * 100 \%,
$$

where $T_{k i}$ is the EDR of the $k^{\text {th }}$ direction at the $i^{\text {th }}$ mode. When the value is $100 \%$, the CVIS is completely decoupled in this mode; that is, vibration occurs only in one direction. Practically, it is difficult to achieve the complete decoupling of one CVIS, so in this research, the EDR in the main vibration direction is greater than $90 \%$, and the EDR in other directions is greater than $80 \%$. 
2.2. Optimization Procedure Based on EDR. According to the state of art and aforementioned research review, it is conventional to select EDR as the objective function for the optimization of CVIS. Therefore, in the next section, the optimization procedure based on EDR is elaborated.

2.2.1. Objective Function. Owning to that the influence degree of each DoF to the isolation performance of CVIS is different, the objective function can be expressed by weighting the EDR of the main mode shapes of each mode:

$$
F_{i}(x)=\min \left\{\sum_{i=1}^{6} \alpha_{i}\left[100 \%-\max \left(T_{k i}\right)\right]\right\},
$$

where $\alpha_{i}$ represents the weighting coefficient corresponding to the frequency of the $i^{\text {th }}$ mode, and $T_{k i}$ represents the EDR of the $k^{\text {th }}$ direction of the $i^{\text {th }}$ mode.

2.2.2. Design Variables. Due to the structural limitations of air conditioning, in this paper, the three-dimensional stiffness is taken as the design variable. Considering that the vibration isolation element is an axisymmetric structure, the design variables are $x=\left[k_{u 1}, k_{w 1}, k_{u 2}, k_{w 2}, k_{u 3}, k_{w 3}, k_{u 4}, k_{w 4}\right]$.

2.2.3. Constraint Conditions. According to the vibration isolation theory and deformation limitation angle design, the natural frequency distribution constraints and stiffness constraints of vibration isolation elements can be expressed as

$$
\begin{cases}5 \leq f_{i} \leq \frac{48.3}{\sqrt{2}}, & (i=1, \ldots, 6), \\ \left|f_{i+1}-f_{i}\right| \geq 1, \quad(i=1, \ldots, 5), & \\ 0.5 \leq k_{u j} \leq 100, & (j=1, \ldots, 4), \\ 10 \leq k_{w j} \leq 200, & (j=1, \ldots, 4), \\ 3 \leq \frac{k_{w j}}{k_{u j}} \leq 8, & (j=1, \ldots, 4),\end{cases}
$$

where $f_{i}$ is the $i^{\text {th }}$ natural frequency and $k_{u j}$ and $k_{w j}$ are the stiffness of the $U$ and $W$ directions of the $j^{\text {th }}$ vibration isolation element, respectively.

\section{Optimization Analysis and Results}

\subsection{Vibration Analysis of Camper Air-Conditioning System}

3.1.1. Operational Vibration Analysis. To analyze and troubleshoot the vibration and noise characteristics in a camper caused by a compressor, a vibration test is performed under starting, steady-state, and stopping operational conditions according to the GB/T7777-2003 (standard for mechanical vibration measurement to evaluate the compressor displacement). The instruments used mainly include Simcenter Testlab software for vibration measurement and analysis, a SCADAS data acquisition front-end system, PCB vibration accelerometers, and an acoustic microphone. To avoid the influence of background vibration, the vibration measurement point is selected at the position of the camper ceiling corresponding to the compressor system (as shown in Figure 2(a)); a noise measurement point is selected at the position of the virtual ear of the camper's right bed. The microphone placement near the right bed is shown in Figure 2(b).

As the duration times of different conditions are different, the signal acquisition times for the starting, steadystate, and stopping conditions are set to be different ( $24 \mathrm{~s}$, $10 \mathrm{~s}$, and $10 \mathrm{~s}$, respectively). The vibration test results of the response point under three operational conditions are shown in Figure 3.

Figure 3 shows the time domain vibration response of the starting, steady-state, and stopping conditions, and it can be concluded that the vibration amplitude of the negative $Z$ direction at the camper interior surface corresponding to the compressor is the highest. Therefore, to accurately analyze the relevance between the vibration of the compressor and the vibration response in the camper, the vibration colormap and the time slice vibration curves of the negative $Z$ direction at the camper interior surface corresponding to the outer compressor under three operational conditions are analyzed, as shown in Figures 4 to 6 .

It can be seen in Figure 5 that there are three obvious peaks for the vibration response in the camper under steadystate conditions: $49.2 \mathrm{~Hz}, 100 \mathrm{~Hz}$, and $300 \mathrm{~Hz}$. According to the excitation characteristics of the compressor, the internal eccentric mass rotor generates an unbalanced inertial force [4] during the rotation, and the frequency is the same as the operational frequency under steady-state conditions $[4,5,17]$. The power frequency of the motor is $50 \mathrm{~Hz}$. According to the characteristics of the internal electromagnetic force of the motor in the air gap magnetic field, the vibration at $100 \mathrm{~Hz}$ and $300 \mathrm{~Hz}$ is harmonic oscillation caused by the radial electromagnetic force, which exists at all three operational conditions.

It can be seen in Figure 4 (vibration response under the starting condition) and Figure 6 (vibration response under the stopping condition) that in addition to the obvious peaks at $49.6 \mathrm{~Hz}, 99.6 \mathrm{~Hz}$, and $300 \mathrm{~Hz}$, serious vibration peaks also occur at $6.8 \mathrm{~Hz}(7.2 \mathrm{~Hz}$ in Figure 6(b)), $22.8 \mathrm{~Hz}$ (under starting conditions), and $22.00 \mathrm{~Hz}$ (under stopping conditions). It can be seen in the colormap that the vibration peaks at $7.2 \mathrm{~Hz}$ only appear momentarily during the compressor starting and stopping process and not the steady-state condition.

Therefore, it can be assumed that the serious vibrations at $7.2 \mathrm{~Hz}$ and $22.0 \mathrm{~Hz}$ are caused by the resonance of rotational excitation frequencies and natural frequencies of the compressor during the acceleration or deceleration of rotary speed under the starting and stopping processes.

3.1.2. Modal Identification Analysis of CVIS. To further study the cause of the transient vibration peaks at $7.2 \mathrm{~Hz}$ or $6.8 \mathrm{~Hz}, 22.8 \mathrm{~Hz}$, or $22.00 \mathrm{~Hz}$ under the starting and stopping 


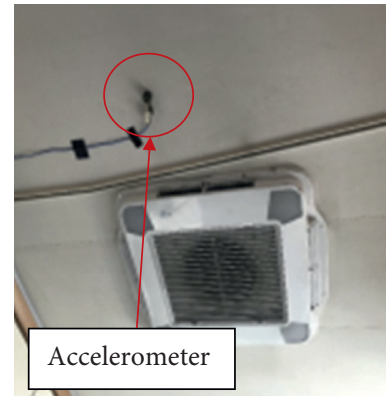

(a)

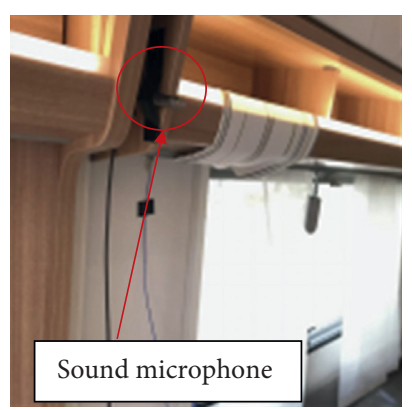

(b)

Figure 2: Accelerometer and microphone placement for the operational test. (a) Accelerometer placement for vibration test. (b) Microphone placement near the right bed.

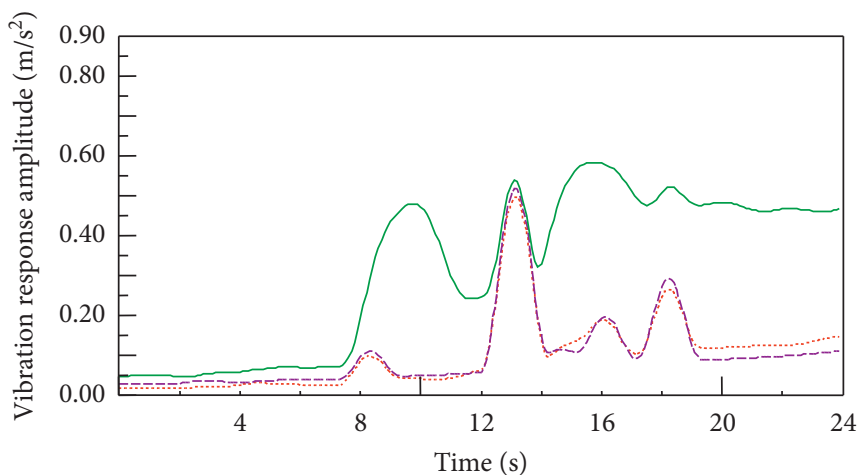

..... Vibration response: $-X$

- - - Vibration response: $+Y$

_ Vibration response: $-Z$

(a)

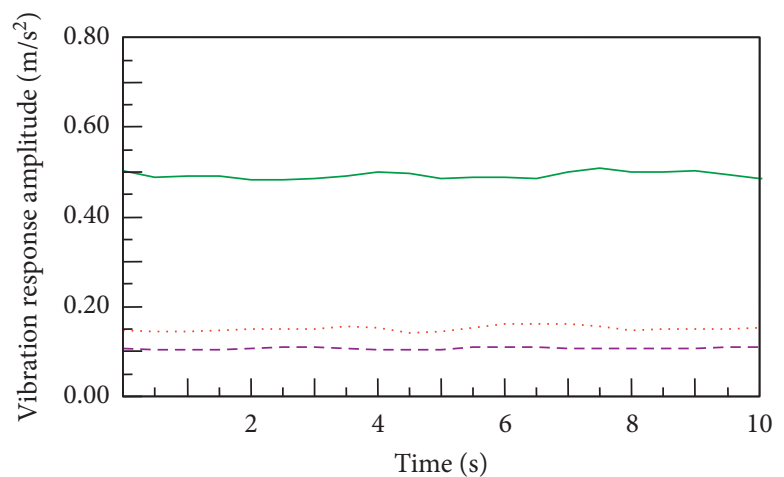

…. Vibration response: $-X$

- - - Vibration response: $+Y$

_ Vibration response: $-Z$

(b)

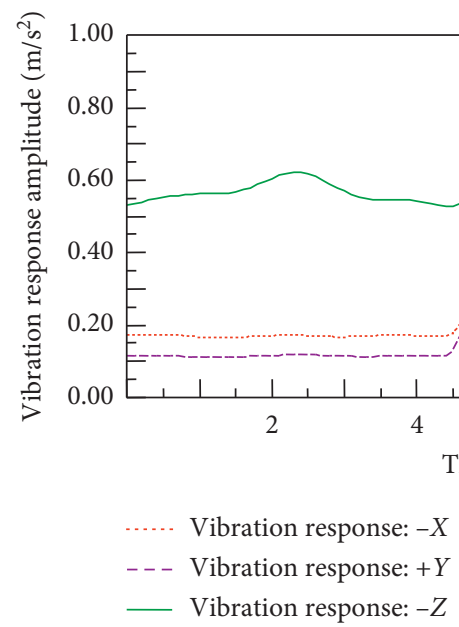

(c)

FIgURE 3: Operational vibration responses in the time domain. (a) Operational vibration response during the starting process. (b) Operational vibration during the steady-state process. (c) Operational vibration response during the stopping process. 


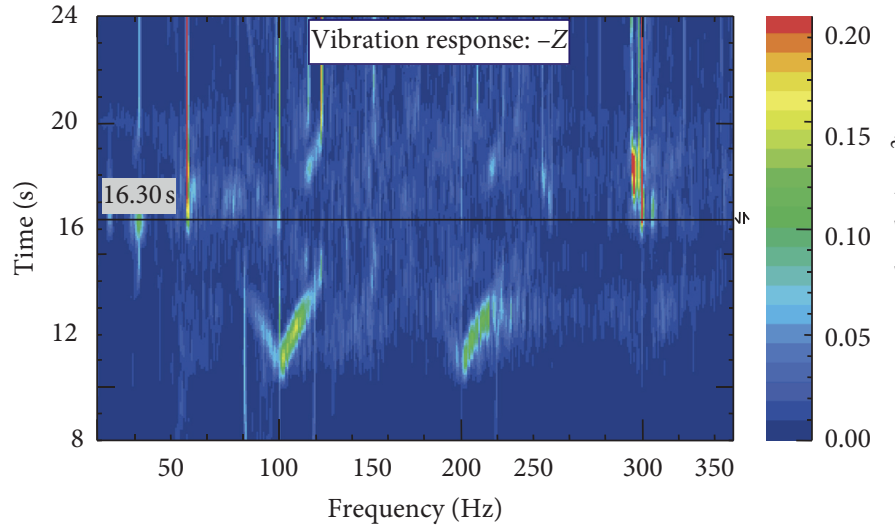

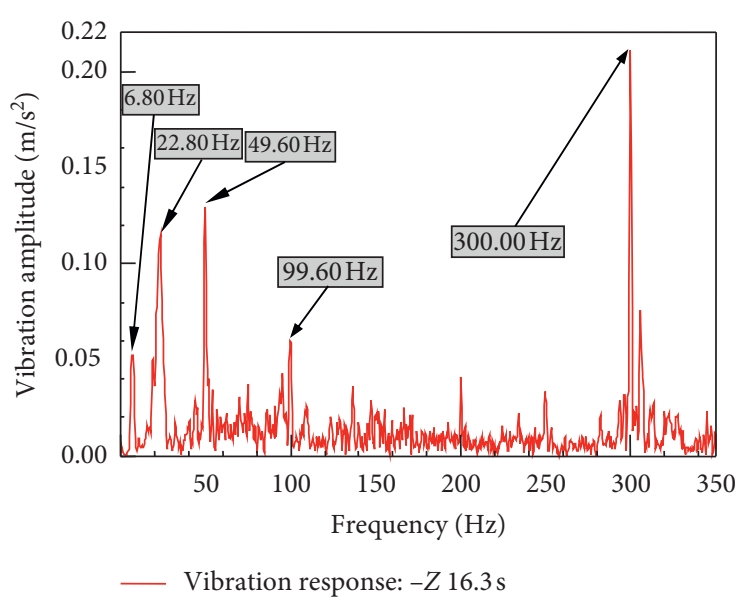

(b)

Figure 4: Vibration colormap and time slice frequency spectrum of the response point under starting conditions. (a) Vibration response colormap. (b) Vibration frequency spectrum at $16.3 \mathrm{~s}$ time slice.

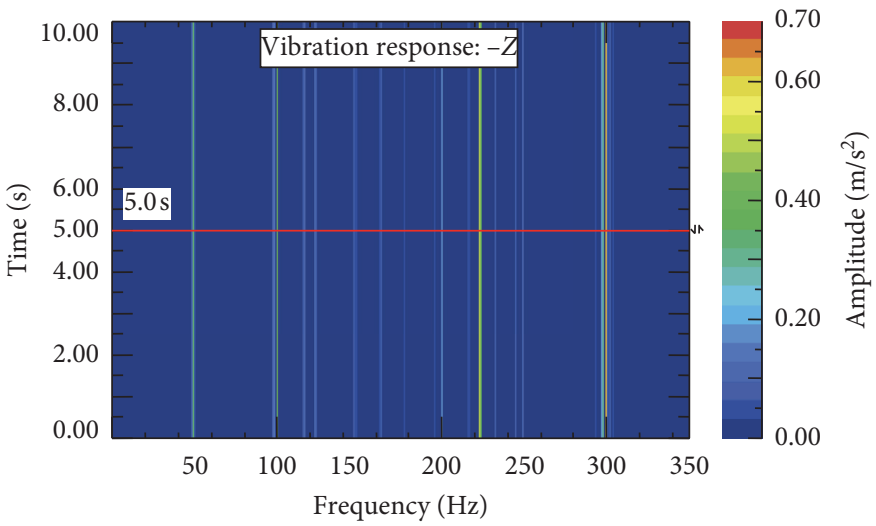

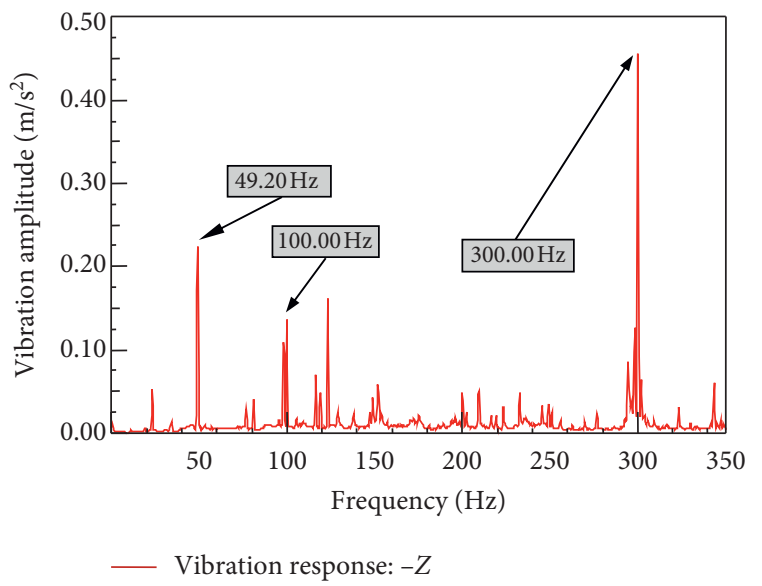

(b)

Figure 5: Vibration colormap and time slice frequency spectrum of the response point under steady-state conditions. (a) Vibration response colormap. (b) Vibration frequency spectrum at $5.0 \mathrm{~s}$ time slice.

conditions of the camper, an operational modal test is necessary to validate the above supposition. Based on an optimal accelerometer placement by the modal kinetic energy method [18-20], the multiple reference point least multiplied complex frequency domain method [21, 22] is adopted to identify the modal parameters by the hammer impact modal test with the reasonable of excitation points and response points and frequency bandwidth determination $[23,24]$. Due to space limitations, only the modal frequencies and mode shapes of the translational $Z$ and rotational $X$ directions of the CVIS are given, as shown in Table 1.

From the modal identification results in Table 1, within the margin of error, the natural frequencies of the translational $Z$ direction $(7.18 \mathrm{~Hz})$ and rotational $X$ direction $(22.44 \mathrm{~Hz})$ are the same as the frequencies of the vibration peaks during the start and stop conditions of the response point in the camper. It can be concluded that the violent vibration during starting and stopping conditions is caused by the resonance of the compressor excitation frequency with its natural frequency of translational $Z$ and rotational $X$ directions.

\subsection{Optimization Analysis of CVIS}

3.2.1. Stiffness, Natural Frequencies, and EDR of CVIS before Optimization. In this research, the compressor mass is $14.35 \mathrm{~kg}$, and the rotational inertia and inertia product parameters are shown in Table 2. By setting the back face center of the compressor cylinder as the coordinate origin, the horizontal direction from cylinder to motor in compressor as the $X$ axis, the horizontal direction from the compressor to the reservoir as the $Y$ axis, and the third axis confirmed by right-hand rule as $Z$ axis, the reference 


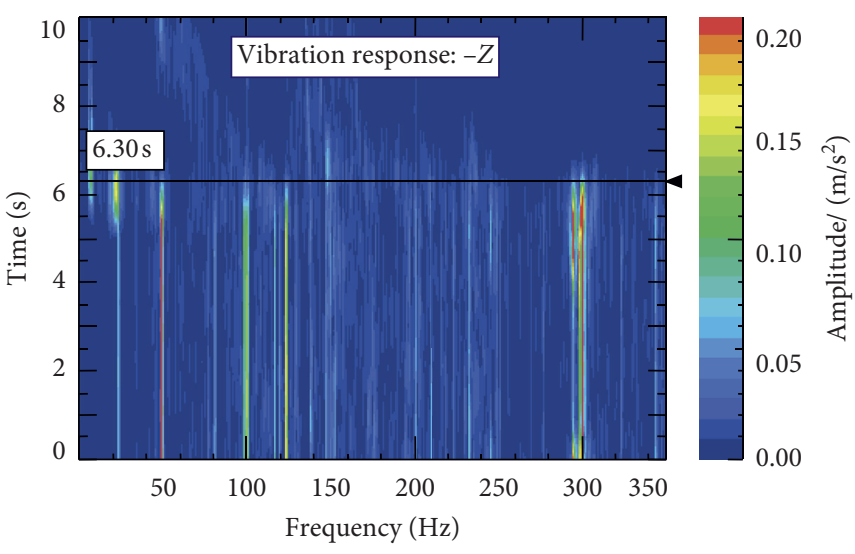

(a)

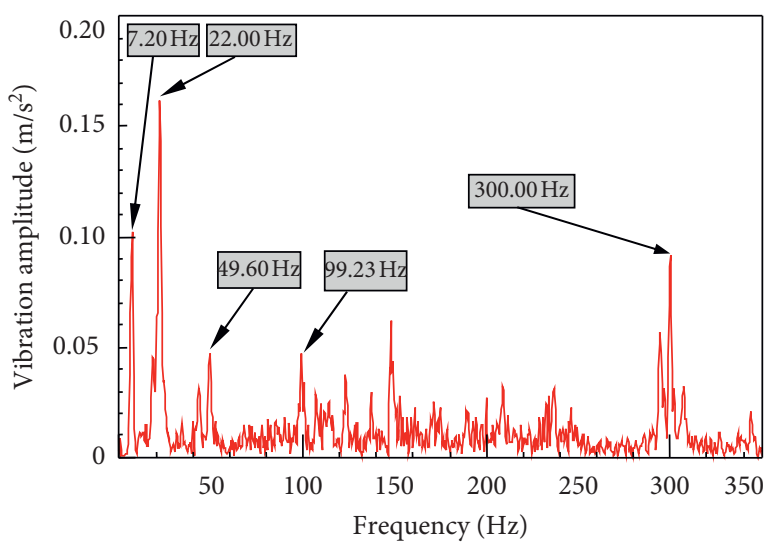

- Vibration response: $-Z 16.3 \mathrm{~s}$

(b)

FIGURE 6: Vibration colormap and time slice frequency spectrum of the response point under stopping conditions. (a) Vibration response colormap. (b) Vibration frequency spectrum at $6.3 \mathrm{~s}$ time slice.

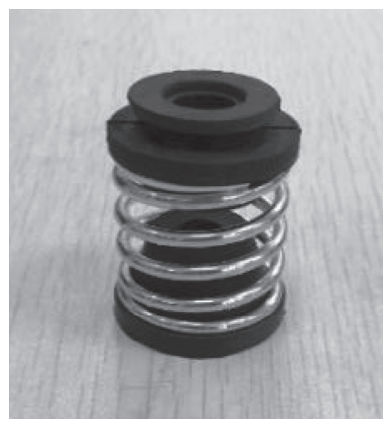

(a)

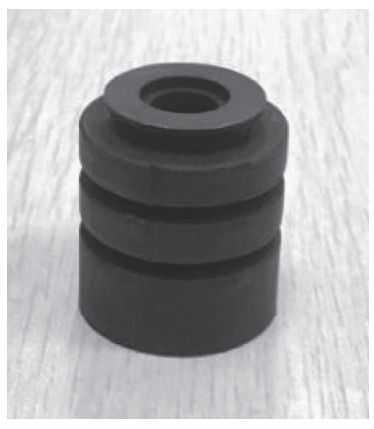

(b)

Figure 7: The vibration isolation element structure pre/postoptimization. (a) Preoptimized element structure. (b) Postoptimized element structure.

TABle 1: Modal characteristics of CVIS.

\begin{tabular}{lll}
\hline Orders & Natural frequencies $(\mathrm{Hz})$ & Modes \\
\hline $4^{\text {th }}$ & $7.18 \mathrm{~Hz}$ & Mranslational $Z$ \\
$6^{\text {th }}$ & Rotational $X$
\end{tabular}


TABLE 2: Rotational inertia and inertial product of the compressor $\left(\mathrm{kg} * \mathrm{~m}^{2}\right)$.

\begin{tabular}{lccccc}
\hline$I_{x x}$ & $I_{y y}$ & $I_{z z}$ & $I_{x y}$ & $I_{y z}$ & $I_{z x}$ \\
\hline 0.0275 & 0.0807 & 0.0777 & -0.0010 & -0.0001 & 0.0062 \\
\hline
\end{tabular}

coordinate system is established. The coordinates for the installation position of the vibration isolation element under the compressor reference coordinate system are shown in Table 3. The three-dimensional stiffness of the vibration isolation element is shown in Table 4.

The natural frequencies and EDR are solved by MAT$\mathrm{LAB}$, the results of which are shown in Table 5 .

The EDR of translational $Z$ and rotational $X$ directions is the main concern for the CVIS. It can be seen in Table 5 that the EDR of the rotational $X$ direction is only $83 \%$, which is less than the $90 \%$ required in practical engineering. There is also vibration coupling to a certain degree in other directions. Therefore, it is necessary to further optimize the CVIS. To analyze the cause of the vibration and noise in the compressor system, an operational vibration test and modal identification are carried out in the next section. Here, note that it is the inevitable simulation error that causes the minus EDR of rotational $X$ of $5^{\text {th }}$ order marked red. Usually, all the EDRs are not less than zero.

3.2.2. Stiffness, Natural Frequencies, and EDR of CVIS after Optimization. For the EDR optimization in this paper, the EDRs of rotational $X$ and translational $Z$ are the key factors; therefore, the weighting coefficient of all the six DoFs in equation (4) is set as $0.1,0.1,0.3,0.3,0.1$, and 0.1 .The EDR of the CVIS is optimized by using a genetic algorithm in MATLAB. The element stiffness, natural frequencies, and decoupling degree of the CVIS after optimization are shown in Tables 6 and 7. Then, the stiffness and strength of the vibration isolation element are verified by finite element simulation.

It can be concluded in Table 6 that after optimization, the stiffness of the $W$ direction increases, and the stiffness of the $U$ and $V$ directions decreases, which is more beneficial for controlling the vibration isolation of the $W$ direction.

Due to the obvious resonance peaks under the starting and stopping conditions of the compressor, the vibration isolation element is redesigned as a pure rubber structure with large damping, whose structure before and after optimization is shown in Figure 7.

Comparing the results in Tables 5 and 7 , it can be concluded that the EDRs of the translational $Z$ direction and rotational $X$ direction after optimization are improved to $99.81 \%$ and $98.68 \%$, respectively; the EDRs of other directions are also greater than $80 \%$; therefore, the system vibration coupling is significantly reduced.

To compare the natural frequencies and EDR of the CVIS between preoptimization and postoptimization, the comparison results are shown in Tables 8 and 9, respectively.

It can be seen in Table 8 that the natural frequencies of the optimized CVIS range between $5 \mathrm{~Hz}$ and $24 \mathrm{~Hz}$, and the maximum natural frequency is much smaller than $1 / \sqrt{2}$
TABLE 3: Installation location of vibration isolation elements ( $\mathrm{mm})$.

\begin{tabular}{lccc}
\hline Vibration isolation element location & $X$ & $Y$ & $Z$ \\
\hline Front left & 227.54 & 74.30 & -64.28 \\
Front right & 227.54 & -74.30 & -64.28 \\
Rear left & 46.64 & 74.30 & -64.28 \\
Rear right & 46.64 & -74.30 & -64.28 \\
\hline
\end{tabular}

TABLE 4: Three-dimensional stiffness of the vibration isolation element of preoptimization $(\mathrm{N} / \mathrm{mm})$.

\begin{tabular}{lccc}
\hline Vibration isolation element location & $U$ & $V$ & $W$ \\
\hline Front left & 15 & 15 & 6.75 \\
Front right & 15 & 15 & 6.75 \\
Rear left & 15 & 15 & 6.75 \\
Rear right & 15 & 15 & 6.75 \\
\hline
\end{tabular}

TABle 5: Natural frequencies (Hz) and EDR (\%) of the CVIS of preoptimization.

\begin{tabular}{lcccccc}
\hline Order & 1 & 2 & 3 & 4 & 5 & 6 \\
\hline Frequency & 5.56 & 5.61 & 6.97 & 15.17 & 16.50 & 21.67 \\
Translational $X$ & 19.6 & 38.80 & 4.70 & 36.76 & 0.28 & 0.01 \\
Translational $Y$ & 53.37 & 29.10 & 0.19 & 0.03 & 0.65 & 16.66 \\
Translational $Z$ & 3.42 & 3.10 & 93.39 & 0.09 & 0.00 & 0.00 \\
Rotational $X$ & 11.39 & 5.73 & 0.03 & 0.02 & -0.16 & 83.00 \\
Rotational $Y$ & 12.25 & 23.19 & 1.69 & 62.44 & 0.40 & 0.03 \\
Rotational $Z$ & 0.11 & 0.09 & 0.00 & 0.65 & 98.84 & 0.31 \\
\hline
\end{tabular}

TABLe 6: Three-dimensional stiffness of the vibration isolation element of postoptimization $(\mathrm{N} / \mathrm{mm})$.

\begin{tabular}{lccc}
\hline Vibration isolation element location & $U$ & $V$ & $W$ \\
\hline Front left & 3.6 & 3.6 & 24.5 \\
Front right & 3.6 & 3.6 & 24.5 \\
Rear left & 9.2 & 9.2 & 20.0 \\
Rear right & 9.2 & 9.2 & 20.0 \\
\hline
\end{tabular}

times the compressor's excitation frequency, which meets the requirement of vibration isolation. The minimum natural frequency is greater than $5 \mathrm{~Hz}$, which avoids resonance with lowfrequency excitation from the road surface and resonance with modes of the camper body. In addition, the natural frequency interval between the translational $Z$ direction and rotational $X$ direction is $11.42 \mathrm{~Hz}$, and the minimum interval between each natural frequency is increased from $0.05 \mathrm{~Hz}$ to $0.75 \mathrm{~Hz}$, which avoids resonance among vibration modes with a larger possibility and reduces the vibration resonance bandwidth.

It can be seen in Table 9 that the EDRs of all DoFs in the CVIS after optimization have been significantly improved and all of which exceed $90 \%$. The EDRs of translational $Z$ and rotational $X$ increased from $93.39 \%$ to $83.00 \%$ to $99.79 \%$ and $98.66 \%$, respectively, which means that the vibration coupling phenomenon is improved and the vibration isolation performance is enhanced. 
U, U3 (CSYS-1)

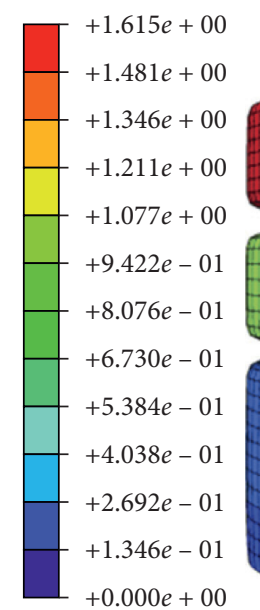<smiles>[Y][Y][3H]</smiles>

U, U3 (CSYS-1)
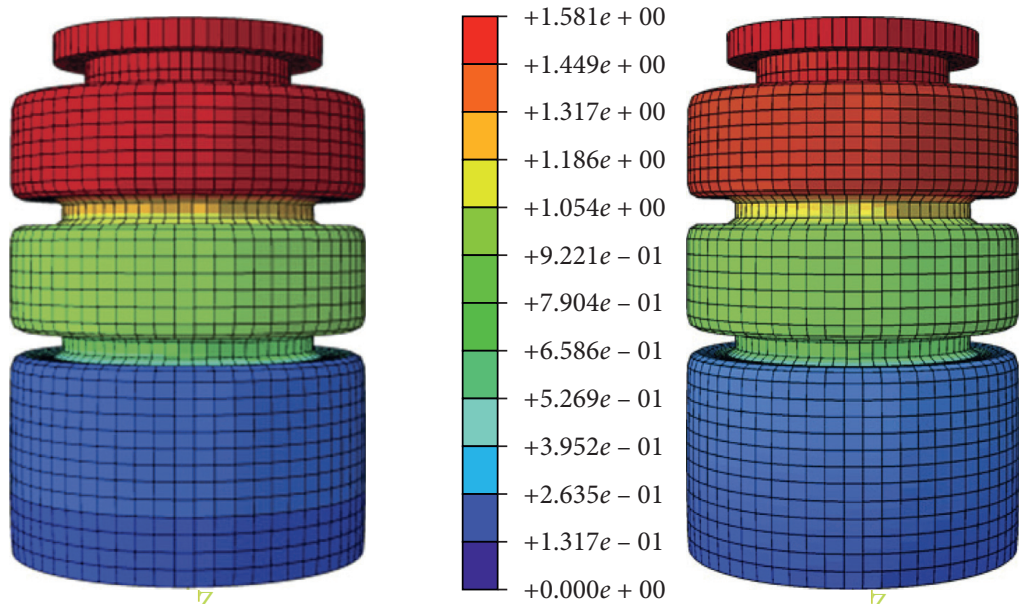

(b)

FIGURE 8: Displacement simulation results of the front and rear vibration isolation elements. (a) Front vibration isolation rubber. (b) Rear vibration isolation rubber.

TABLE 7: Natural frequencies (Hz) and EDR (\%) of the CVIS-postoptimization.

\begin{tabular}{|c|c|c|c|c|c|c|}
\hline Orders & 1 & 2 & 3 & 4 & 5 & 6 \\
\hline Frequencies & 5.38 & 6.15 & 11.30 & 12.53 & 16.45 & 23.93 \\
\hline Translational $X$ & 0.08 & 96.73 & 0.00 & 0.01 & 3.17 & 0.00 \\
\hline Translational $Y$ & 90.97 & 0.09 & 7.26 & 0.02 & 0.00 & 1.67 \\
\hline Translational $Z$ & 0.00 & 0.00 & 0.07 & 99.79 & 0.11 & 0.03 \\
\hline Rotational $X$ & 1.43 & 0.00 & -0.19 & 0.02 & 0.06 & 98.67 \\
\hline Rotational $Y$ & 0.00 & 3.18 & 0.01 & 0.10 & 96.66 & 0.07 \\
\hline Rotational $Z$ & 7.52 & 0.00 & 92.85 & 0.06 & 0.01 & -0.43 \\
\hline
\end{tabular}

TABLE 8: Natural frequency comparison of CVIS between preoptimization and postoptimization.

\begin{tabular}{|c|c|c|c|c|c|c|c|}
\hline Order & & $1^{\text {st }}$ & $2^{\text {nd }}$ & $3^{\text {rd }}$ & $4^{\text {th }}$ & $5^{\text {th }}$ & $6^{\text {th }}$ \\
\hline \multirow{2}{*}{ Natural frequencies } & Preoptimization & 5.56 & 5.61 & 6.97 & 15.17 & 16.50 & 21.69 \\
\hline & Postoptimization & 5.38 & 6.15 & 11.30 & 12.53 & 16.45 & 23.93 \\
\hline
\end{tabular}

TABLE 9: EDR comparison of CVIS between preoptimization and postoptimization.

\begin{tabular}{lccccccc}
\hline $\begin{array}{l}\text { DoF with principal vibration } \\
\text { energy }\end{array}$ & Translational $X$ & Translational $Y$ & Translational $Z$ & Rotational $X$ & Rotational $Y$ & Rotational $Z$ \\
\hline \multirow{2}{*}{ EDR (\%) } & Preoptimization & 38.80 & 53.37 & 93.39 & 83.00 & 62.44 \\
& Postoptimization & 96.73 & 90.97 & 99.79 & 98.67 & 96.66 \\
\hline
\end{tabular}

3.2.3. Optimized Stiffness and Strength Verification. To verify whether the stiffness of the vibration isolation element after theoretical optimization concurs with the actual stiffness of the vibration isolation element, the stiffness and strength of the vibration isolation element are confirmed by finite element simulation.
(1) Stiffness Verification. The stiffness of the vibration isolation element is simulated by using ABAQUS, and the vertical displacement simulation results are shown in Figure 8.

It can be seen in Figure 8 that the maximum vertical displacements of the front and rear vibration isolation 


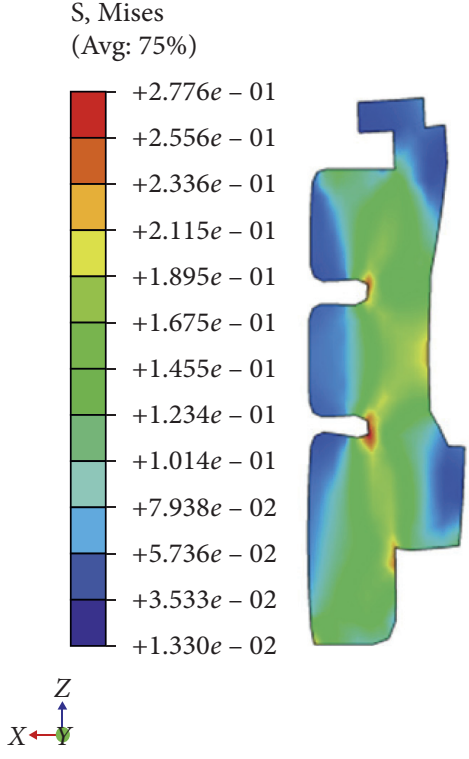

(a)
S, Mises

(Avg: 75\%)
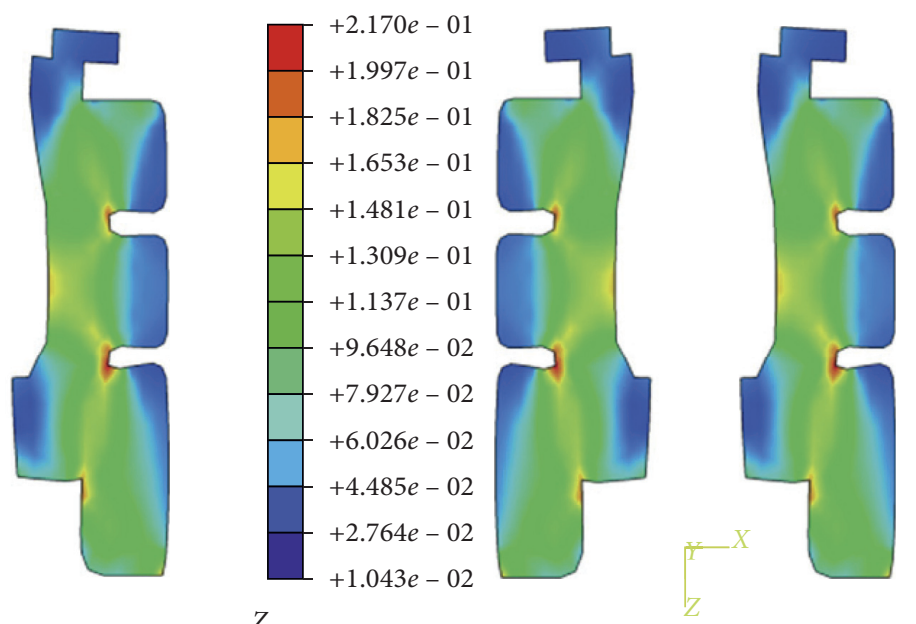

(b)

Figure 9: The section stress map of the front and rear vibration isolation elements. (a) Front vibration isolation rubber. (b) Rear vibration isolation rubber.

U, U3 (CSYS-1)

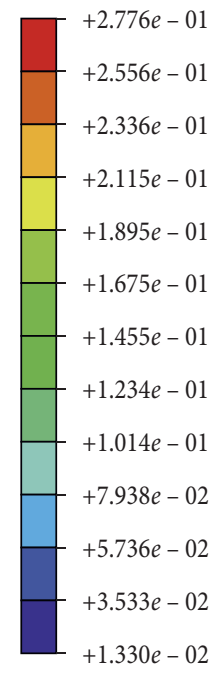

$X \stackrel{+}{Z}$

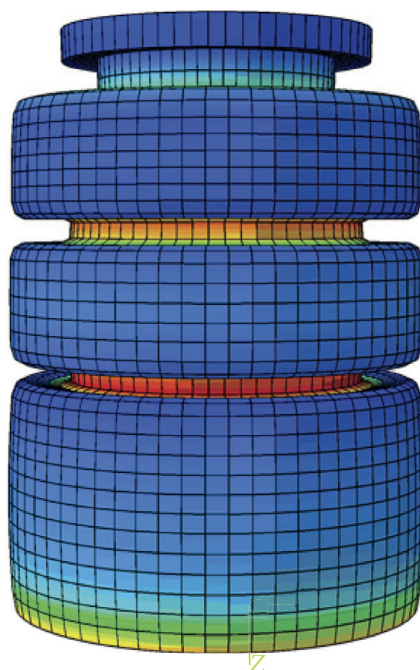

(a)
U, U3 (CSYS-1)

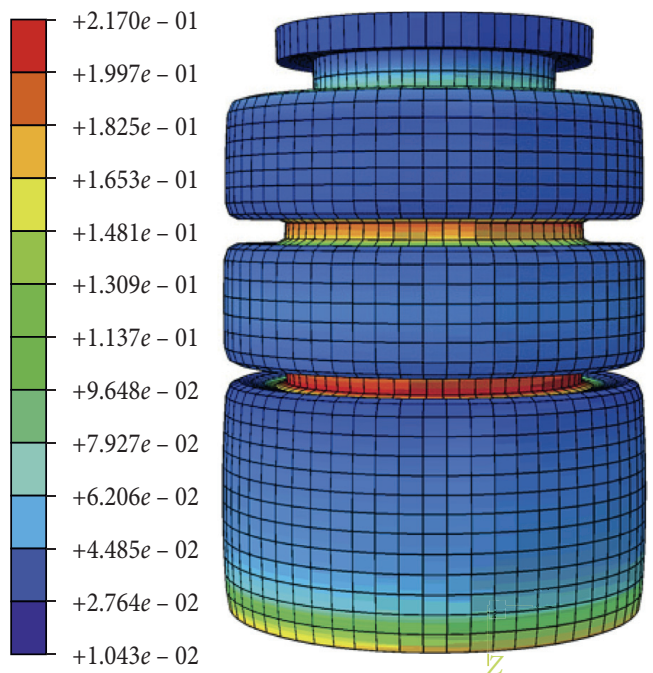

$X \stackrel{+}{Z}$

(b)

Figure 10: Overall stress map of the front and rear vibration isolation elements. (a) Front vibration isolation rubber. (b) Rear vibration isolation rubber.

elements are $1.615 \mathrm{~mm}$ and $1.581 \mathrm{~mm}$, and the compression deformation ratios are $4.68 \%$ and $4.58 \%$; the vertical stiffness values are $24.53 \mathrm{~N} / \mathrm{mm}$ and $19.45 \mathrm{~N} / \mathrm{mm}$, respectively, with optimization value errors of $0.12 \%$ and $2.85 \%$, respectively. The results show that the physically processed vibration isolation elements meet the stiffness requirement.
(2) Strength Verification. The strength verification is simulated by using ABAQUS, and the corresponding section stress map, overall stress map, and vertical displacement map are obtained, as shown in Figures 9 to 11.

It can be seen in Figures 9 and 10 that the maximum equivalent stresses of the front and rear vibration isolation elements are $0.2776 \mathrm{MPa}$ and $0.217 \mathrm{MPa}$, respectively, and 


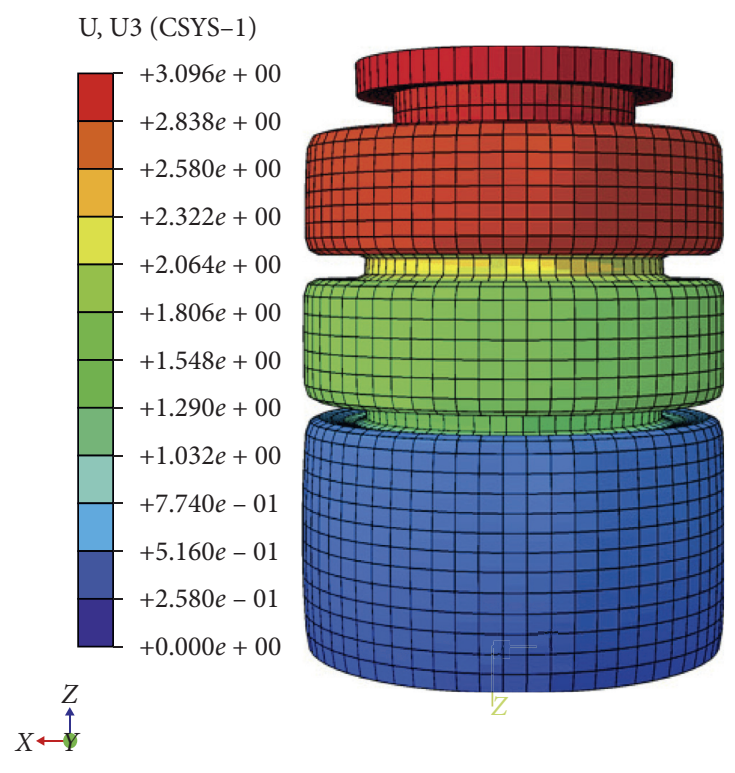

(a)

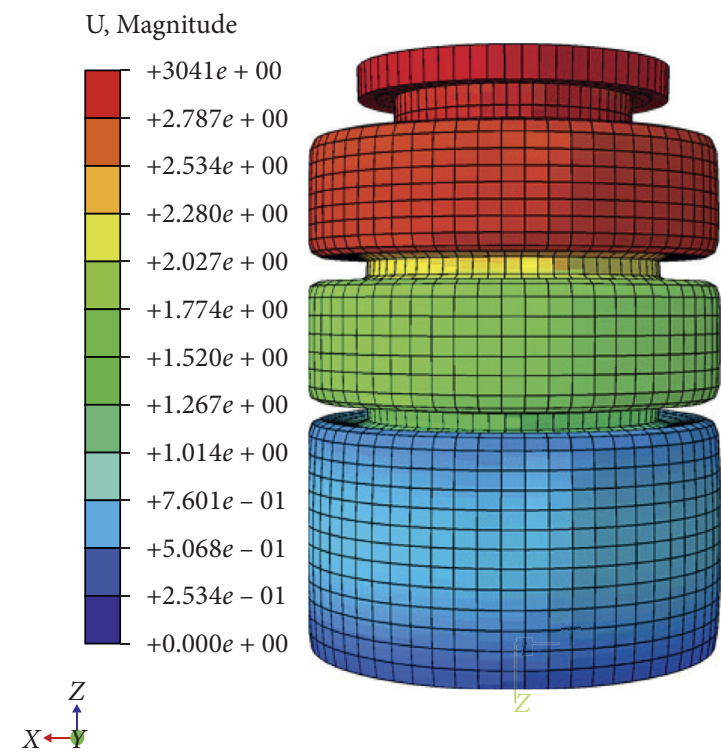

(b)

Figure 11: Vertical displacement map of the front and rear vibration isolation elements. (a) Front vibration isolation rubber. (b) Rear vibration isolation rubber.

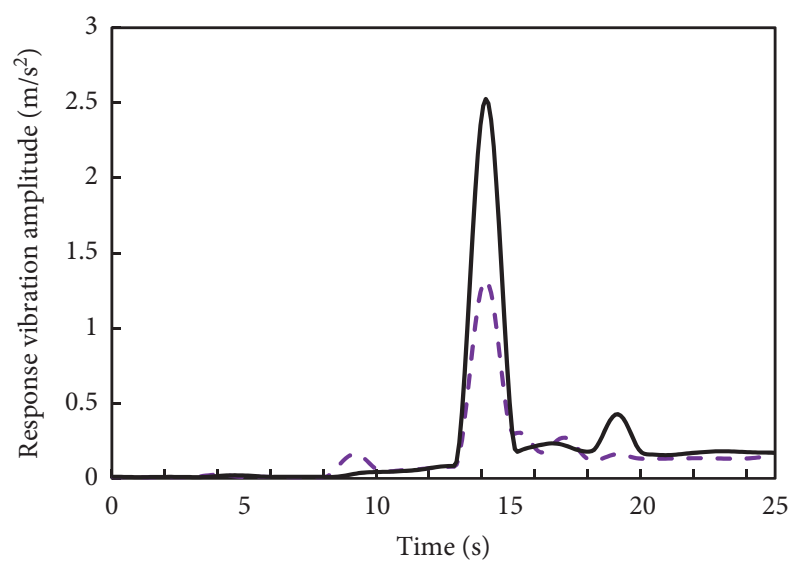

--- $X$ direction_postoptimization

— X direction_preoptimization

(a)

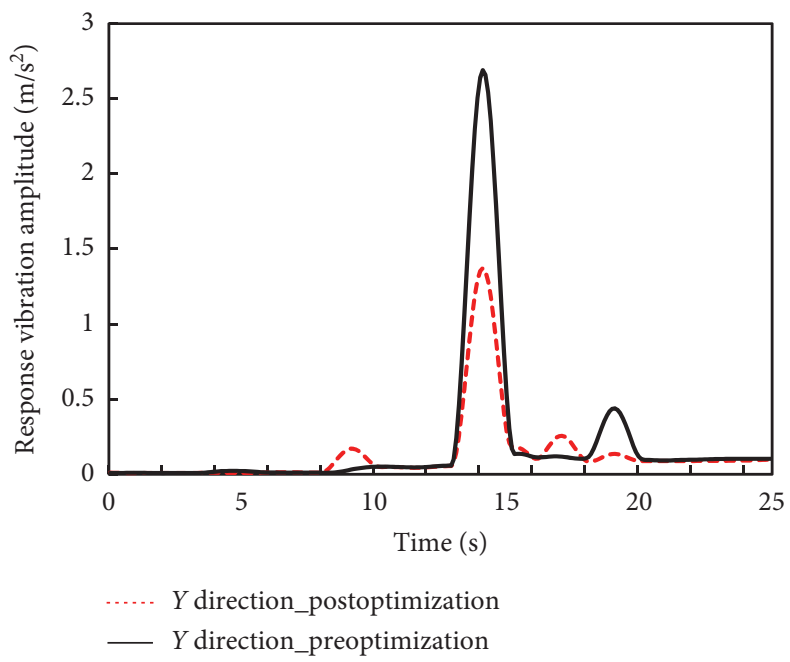

(b)

Figure 12: Continued. 


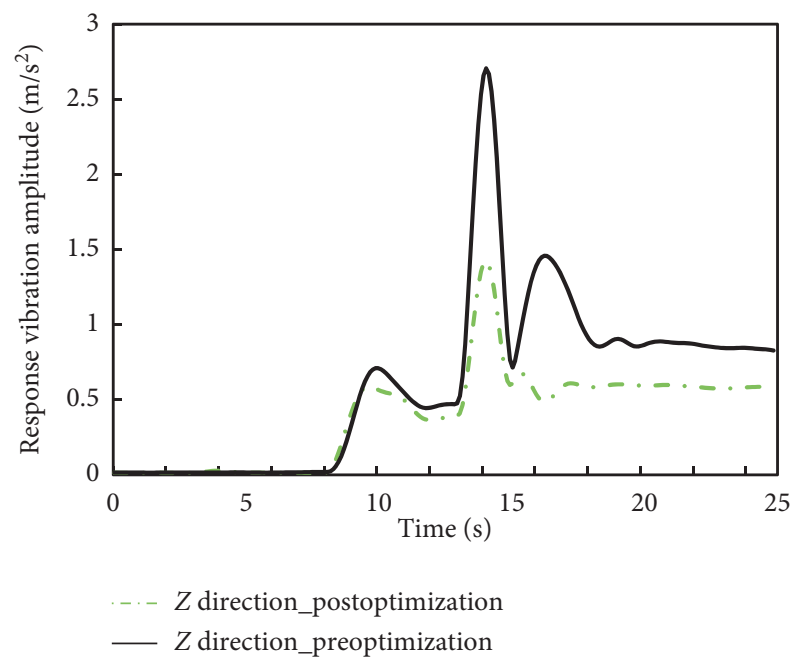

(c)

FIGURE 12: The time domain vibration of the response point in the camper under preoptimized and postoptimized starting conditions. (a) Vibration comparison in the $X$ direction. (b) Vibration comparison in the $Y$ direction. (c) Vibration comparison in the $Z$ direction.

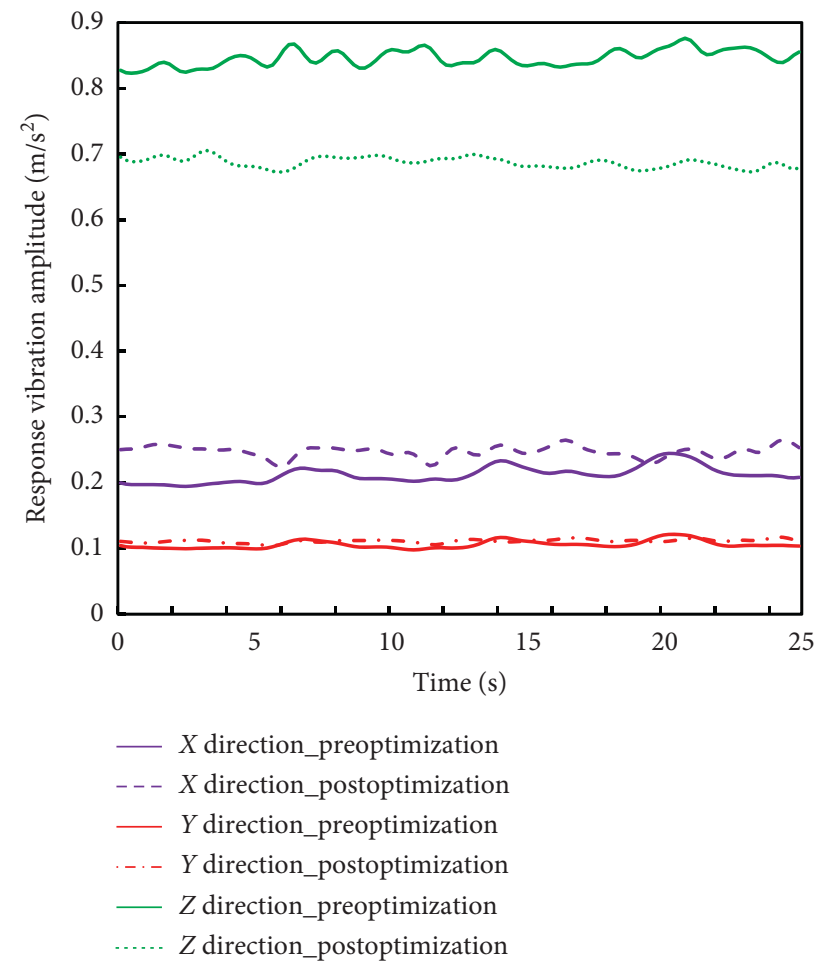

FIGURE 13: The time domain vibration of the response point in the camper under preoptimized and postoptimized steady-state conditions.

they all occur at the outer surface of the second $U$-shaped groove. The allowable stress of native rubber is $1.5 \mathrm{MPa}$. Obviously, the front and rear vibration isolation elements meet the allowable stress requirements.

Figure 11 shows that the maximum vertical displacements of the front and rear vibration isolation elements are $3.096 \mathrm{~mm}$ and $3.041 \mathrm{~mm}$, respectively, and the compressed deformation ratios of the two elements are $8.97 \%$ and $8.80 \%$, both of which meet the maximum allowable deformation ratio of less than $10 \%$.

\section{Optimization Validation}

To verify the optimization benefit to improving the vibrations of air-conditioning systems, the optimization effect is validated by a camper operational vibration and noise test 


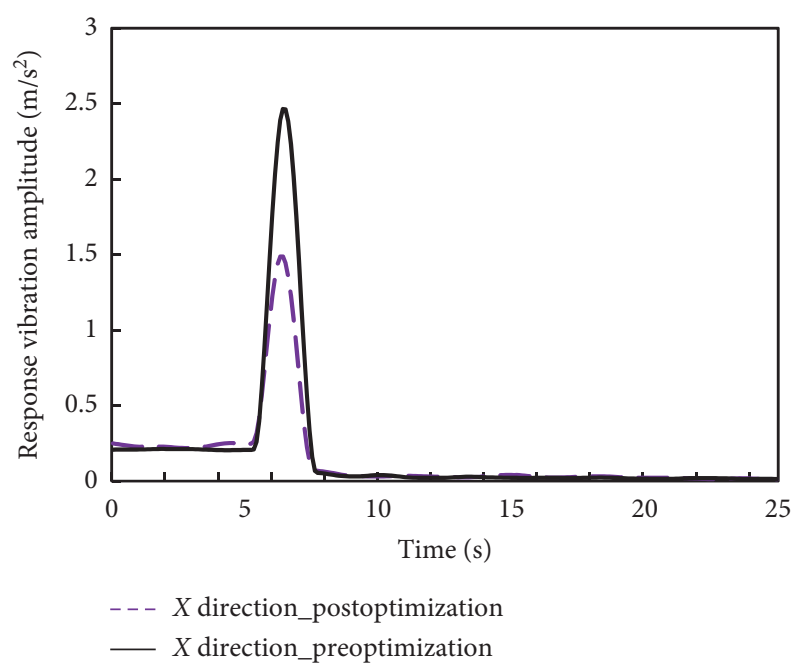

(a)

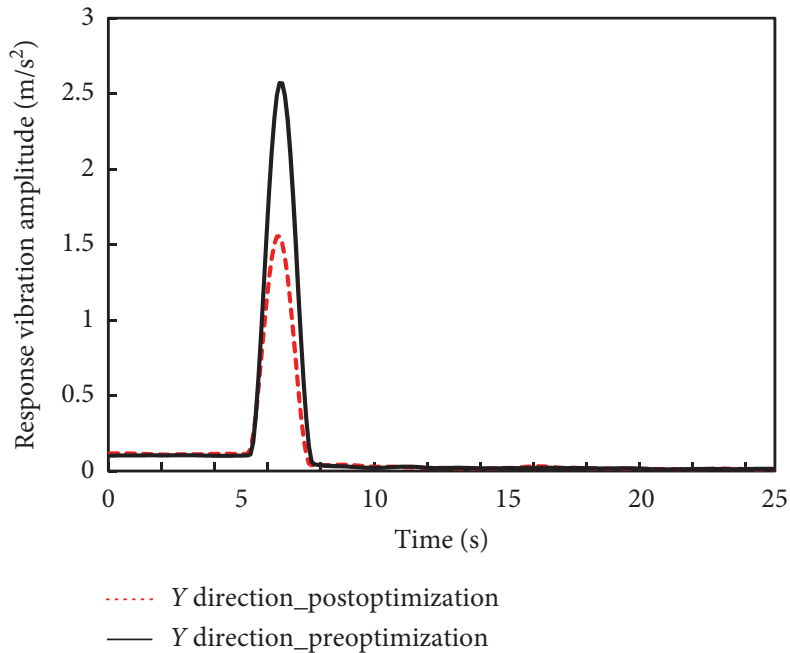

(b)

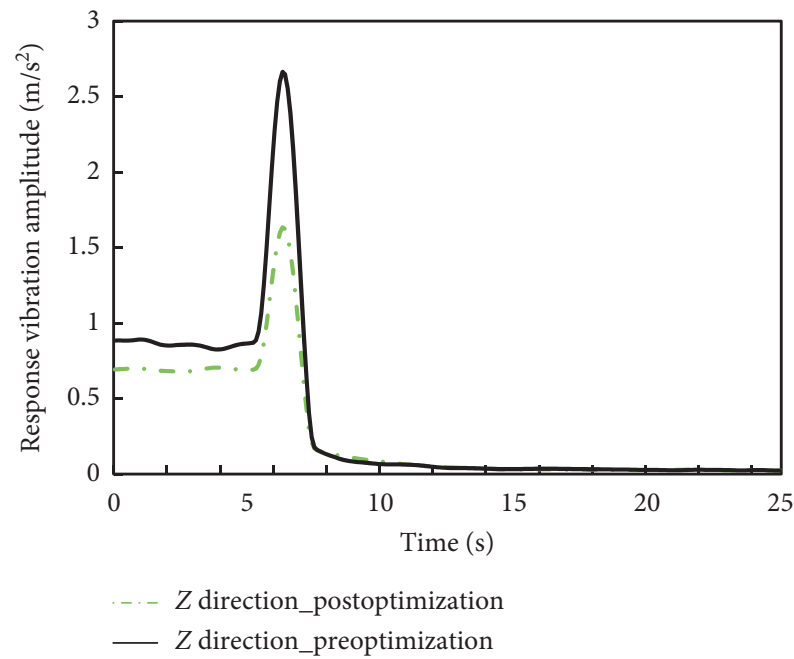

(c)

Figure 14: The time domain vibration of the response point in the camper under preoptimized and postoptimized steady-state and stopping conditions. (a) Vibration comparison in the $X$ direction. (b) Vibration comparison in the $Y$ direction. (c) Vibration comparison in the $Z$ direction.

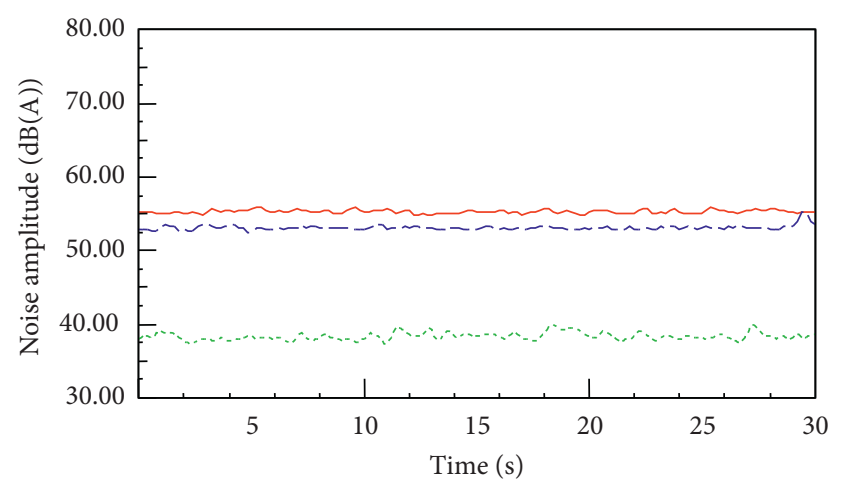

…. Noise level_background
_ Noise level_preoptimization
- - - Noise level_postoptimization

FIGURE 15: The preoptimization and postoptimization noise in the time domain. under starting, steady-state, and stopping conditions both before and after optimization with the same test environment at the same time. For the preoptimization test in this section, a period of time has elapsed since the vibration test for troubleshooting the problem in Section 3, and the test conditions and environments for these two tests are very different. Therefore, it is the comparison between the preoptimization and postoptimization of this section and not the comparison between the postoptimization in this section and the test results in Section 3, which will be used to validate the optimization effect.

4.1. Vibration Response Analysis. The vibration comparison results are shown in Figures 12 to 14, and the noise comparison results are shown in Figure 15.

Under the starting condition of air conditioning in Figure 12, the vibration peak value in the $X$ direction at the response point was reduced from $2.52 \mathrm{~m} / \mathrm{s}^{2}$ to $1.32 \mathrm{~m} / \mathrm{s}^{2}$, 
with a reduction rate of $47.62 \%$; the vibration peak value in the $Y$ direction was reduced from $2.69 \mathrm{~m} / \mathrm{s}^{2}$ to $1.37 \mathrm{~m} / \mathrm{s}^{2,}$ with a reduction rate of $49.07 \%$, and the vibration peak value in the $Z$ direction was reduced from $2.71 \mathrm{~m} / \mathrm{s}^{2}$ to $1.43 \mathrm{~m} / \mathrm{s}^{2}$, with a reduction rate of $47.23 \%$.

It can be seen in Figure 13 that under the steady-state condition of the air-conditioning system, the response vibration is basically unchanged in both the $X$ and $Y$ directions, but the $Z$ direction vibration of interest is reduced by more than $0.13 \mathrm{~m} / \mathrm{s}^{2}$.

It can be seen in Figure 14 that under the stopping condition, the vibration peak value at the response point is reduced from $2.46 \mathrm{~m} / \mathrm{s}^{2}$ to $1.49 \mathrm{~m} / \mathrm{s}^{2}$ in the $X$ direction, with a reduction rate of $39.43 \%$, from $2.57 \mathrm{~m} / \mathrm{s}^{2}$ to $1.55 \mathrm{~m} / \mathrm{s}^{2}$ in the $Y$ direction, with a reduction rate of $39.68 \%$, and from $2.66 \mathrm{~m} / \mathrm{s}^{2}$ to $1.63 \mathrm{~m} / \mathrm{s}^{2}$ in the $Z$ direction, with a reduction rate of $38.72 \%$.

4.2. Noise Response Analysis. The noise response at the position of the virtual human ear of the right bed in the camper is compared between preoptimization and postoptimization, and the comparison result is shown in Figure 15.

It can be seen in Figure 15 that the noise pressure in the camper is reduced by $2.23 \mathrm{~dB}$ after optimization, indicating that the camper comfort is improved after optimization.

\section{Conclusion and Discussion}

This paper has introduced an optimization procedure of the vibration isolation system based on the energy decoupling method for improving the camper comfort. Based on the operational vibration and noise test, a genetic algorithm is used to optimize the EDR of CVIS and the optimization effectiveness is verified by simulations and operational tests. The conclusions are obtained as follows:

(a) The presented optimization procedure offers a systematical method for the optimization of CVIS for the camper air-conditioning system, which provides an additional strategy reducing vibration and noise level of camper comfort

(b) The operational vibration and noise test and modal identification indicate that the severe vibration in the camper air-conditioning system during starting and stopping is caused by the vibration resonance of the compressor rotational excitation frequencies with the compressor system natural frequencies

(c) The energy decoupling rates of CVIS are optimized by a genetic algorithm, and the stiffness and strength is verified by FEM simulation, of which the analysis results between preoptimization and postoptimization show that the sated optimization method is effective numerically

(d) The redesigned rubber isolation element is tested by the operational test, of which the vibration and noise test results suggest that the camper comfort is noticeably promoted; however, further designing types of isolation elements deserve to be explored

The stated systematical optimization method is effective to enhance the excitation force transmissibility of CASs to reduce vibration and noise in the camper; however, further combined methods are hopeful to be investigated, such as combining all excitation forces simulation of compressors by numerical methods with the optimization methods in this paper, better enhancement of camper comfort is to be obtained. Furthermore, the transfer path contribution of each rubber isolation element to the interested response points in camper should be studied quantitatively, which can be used to determine a better weighting coefficient in equation (4) for better EDR optimization results.

\section{Data Availability}

The data used to support the findings of this study are available from the corresponding author upon request.

\section{Conflicts of Interest}

The authors declare that there are no conflicts of interest regarding the publication of this paper.

\section{Acknowledgments}

This research was supported and acknowledged by the China National Key Research \& Design Projects (2018YFB0106203) associated with Jilin University. The authors would like to express their gratitude to Liuliu Hang for his helpful effort in the operational vibration test.

\section{References}

[1] H.-J. Lee and K.-J. Kim, "Multi-dimensional vibration power flow analysis of compressor system mounted in outdoor unit of an air conditioner," Journal of Sound and Vibration, vol. 272, no. 3-5, pp. 607-625, 2004.

[2] Y. C. Park, "Transient analysis of a variable speed rotary compressor," Energy Conversion and Management, vol. 51, no. 2 , pp. $277-287,2010$

[3] N. Levecque, J. Mahfoud, D. Violette, G. Ferraris, and R. Dufour, "Vibration reduction of a single cylinder reciprocating compressor based on multi-stage balancing," Mechanism and Machine Theory, vol. 46, no. 1, pp. 1-9, 2011.

[4] Z. Wang, X. Yu, F. Liu, Q. Feng, and Q. Tan, "Dynamic analyses for the rotor-journal bearing system of a variable speed rotary compressor," International Journal of Refrigeration, vol. 36, no. 7, pp. 1938-1950, 2013.

[5] J. Antoni, F. Bonnardot, A. Raad, and M. El Badaoui, "Cyclostationary modelling of rotating machine vibration signals," Mechanical Systems and Signal Processing, vol. 18, no. 6, pp. 1285-1314, 2004.

[6] Z. Yi, "Analysis and control of noise radiated from air conditional compressors," Master thesis, Shanghai Jiaotong University, Shanghai, China, 2003.

[7] F. Seve, A. Berlioz, R. Dufour et al., "On the unbalance response of a rotary compressor," in Proceedings of the Fifteenth 
International Compressor Engineering Conference, Purdue University, West Lafayette, IN, USA, July 2000.

[8] S. G. Kim, K. L. Cho, Y. S. Cho et al.,"Reduction of the vibration of a home air conditioner by finite element analysis and homogenization method," in Proceedings of the 15th International Modal Analysis Conference, February 1997, Orlando, FL, USA, 3089: 1739,.

[9] S. Li, K. Wang, and W. Xu, "Research on noise and vibration reduction of compress of conditioner," Noise and Vibration Control, no. 3, pp. 27-30, 2003.

[10] C. Li and J. Zhang, "Vibration isolation optimization of refrigerator compressors based on chaotic particle swarm optimization," Noise and Vibration Control, vol. 38, no. 4, pp. 27-33, 2018.

[11] B. Li and Y. Fan, "Study on the impact of shock absorber on vibration and noise of air-conditioner's outdoor unit," Noise and Vibration Control, vol. 30, no. 2, pp. 45-65, 2010.

[12] Z. Dong, X. Huang, and H. Shen, "Dynamics analysis and optimization on the supporting system of a refrigeration compressor," Journal of Mechanical Strength, no. 2, pp. 205-210, 2006.

[13] F.-M. Wang and L. Song, "Dynamic analysis and test of 2DOF vibration isolation system of a refrigerator compressor," Noise and Vibration Control, vol. 37, no. 3, pp. 92-95, 2017.

[14] X. Liu, "Design of the powertrain mounting system based on vibration control of vehicle level" Ph.D thesis, South China University of Technology, Guangzhou, China, 2016.

[15] Y. Long, "Optimum design of engine mounting system based on improved transfer path analysis" Ph.D thesis, Jilin University, Changchun, China, 2010.

[16] R. Guo and T. Zhang, Automotive Powertrain Mounting System, Press of Tongji University, Shanghai, China, 2013.

[17] G. Ferraris, M. A. Andrianoely, A. Berlioz, and R. Dufour, "Influence of cylinder pressure on the balancing of a rotary compressor," Journal of Sound and Vibration, vol. 292, no. 3-5, pp. 899-910, 2006.

[18] H. Jin, J. Xia, and Y. Q. Wang, "Optimal sensor placement for space modal identification of crane structures based on an improved harmony search algorithm," Journal of Zhejiang University: Science A, vol. 16, no. 6, pp. 464-477, 2015.

[19] A. Schulze, J. Zierath, S.-E. Rosenow et al., "Optimal sensor placement for modal testing on wind turbines," Journal of Physics: Conference Series, vol. 753, no. 7, Article ID 072031, 2016.

[20] B. Peeters, H. Vander Auweraer, P. Guillaume, and J. Leuridan, "The polyMax frequency-domain method: a new standard for modal parameter estimation," Shock and Vibration, vol. 11, Article ID 523692, 2004.

[21] H. Hui, "Applications of the polyMax method in aerocrafts modal parameter estimation,” Aero Weaponry, no. 6, pp. 711, 2010.

[22] P. Guillaume, P. Verboven, S. Vanlanduit et al., in "A polyreference implementation of the least squares complex frequency domain estimator," in Proceedings of the 21th International Modal Analysis Conference, Kissimmee, FL, USA, February, 2003.

[23] F. Xu, C. Li, and T. Jiang, "Optimization of excitation and measurement location in experimental modal test," Journal of Beijing University of Aeronautics and Astronautics, vol. 39, no. 12, pp. 1654-1659, 2013.

[24] D. C. Kammer, "Sensor placement for on-orbit modal identification and correlation of large space structures," Journal of Guidance, Control, and Dynamics, vol. 14, no. 2, pp. 251-259, 1991. 